\title{
A model for non-thermal action of microwave radiation on oxide film/semiconductor structures
}

\author{
O.B. Okhrimenko \\ V. Lashkaryov Institute of Semiconductor Physics, NAS of Ukraine \\ 41, prospect Nauky, 03028 Kyiv, Ukraine \\ Phone: 38(044) 525-62-61; fax: 38(044) 525-83-42; e-mail: olga@isp.kiev.ua
}

\begin{abstract}
A model is considered that explains mechanism of non-thermal action of microwave radiation on the thin $\mathrm{SiO}_{2}\left(\mathrm{TiO}_{2}, \mathrm{Er}_{2} \mathrm{O}_{3}, \mathrm{Gd}_{2} \mathrm{O}_{3}\right)$ film/ $\mathrm{SiC}$ and $\mathrm{SiO}_{2} / \mathrm{GaAs}$ structures. It assumes that the centers of electron-hole recombination are redistributed because of resonance interaction between dislocations of certain length and microwave radiation. As a result, additional bands appear in photoluminescence (PL) spectra of the oxide film/SiC structures or intensities of some bands are redistributed in the PL spectra of the $\mathrm{SiO}_{2} / \mathrm{GaAs}$ structure, as well as optical density of the oxide film/SiC structures changes.
\end{abstract}

Keywords: microwave radiation, dislocation, transmission spectrum, photoluminescence, silicon carbide.

Manuscript received 03.03.14; revised version received 21.07.14; accepted for publication 16.09.14; published online 30.09.14.

\section{Introduction}

It is known that action of microwave radiation on device structures and final products (diodes, transistors, integrated circuits) often leads to their degradation and catastrophic failures. There are literature data indicating the effects of defect gettering and structural relaxation in semiconductor materials induced by microwave radiation. In those cases, attention is paid to non-thermal nature of such actions as well as on processes induced by them at the metal-semiconductor and insulatorsemiconductor interfaces $[1,2]$ that are integral parts of MIS transistor structures. At the same time, there is no model in literature that could unambiguously explain mechanism of non-thermal action on the oxide layer/semiconductor structures.

In this work, a model is proposed that gives consistent explanation for mechanism of non-thermal action of microwave radiation on the thin oxide film $\mathrm{SiO}_{2}$ $\left(\mathrm{TiO}_{2}, \mathrm{Er}_{2} \mathrm{O}_{3}, \mathrm{Gd}_{2} \mathrm{O}_{3}\right) / \mathrm{SiC}$ and $\mathrm{SiO}_{2} / \mathrm{GaAs}$ structures.

\section{Experimental results}

In experimental works [3-7] it was shown that a shortterm microwave annealing of frequency $2.45 \mathrm{GHz}$ leads to increase of optical transmission in thin oxide film/silicon carbide structures (Fig. 1) and appearance of additional bands in photoluminescence (PL) spectra of similar structures (Fig. 2) or redistribution of PL bands intensity, as in $\mathrm{GaAs} / \mathrm{SiO}_{2}$ structures (Fig. 3).

It was shown in $[1,2,8-12]$ that changes in semiconductor defect subsystem under action of microwave radiation may be of thermal as well as nonthermal character. The thermal mechanisms of action of microwave radiation may be classified into three groups for convenience $[8,9]$. One of the possible mechanisms is related to dielectric polarization. Such type of action of microwave field on dielectric materials involves the following processes:

- distortion of electron clouds of separate atoms; generally the electron shells of many-electron atoms (those of high atomic number) are easier deformed (atoms are polarized easier);

- $\quad$ alignment of molecules or structural elements with a constant dipole momentum along the field lines;

- deformation (variation of bond angle and length) of molecules, both with and without dipole moment, under action of a microwave field.

Another mechanism of action of microwave radiation involves free-charge currents that are excited in solids and contribute to heating because of ohmic losses. This mechanism is typical for high-conduction solids. One more mechanism to be accounted for is due to ohmic losses related to eddy currents excited by magnetic fields. 

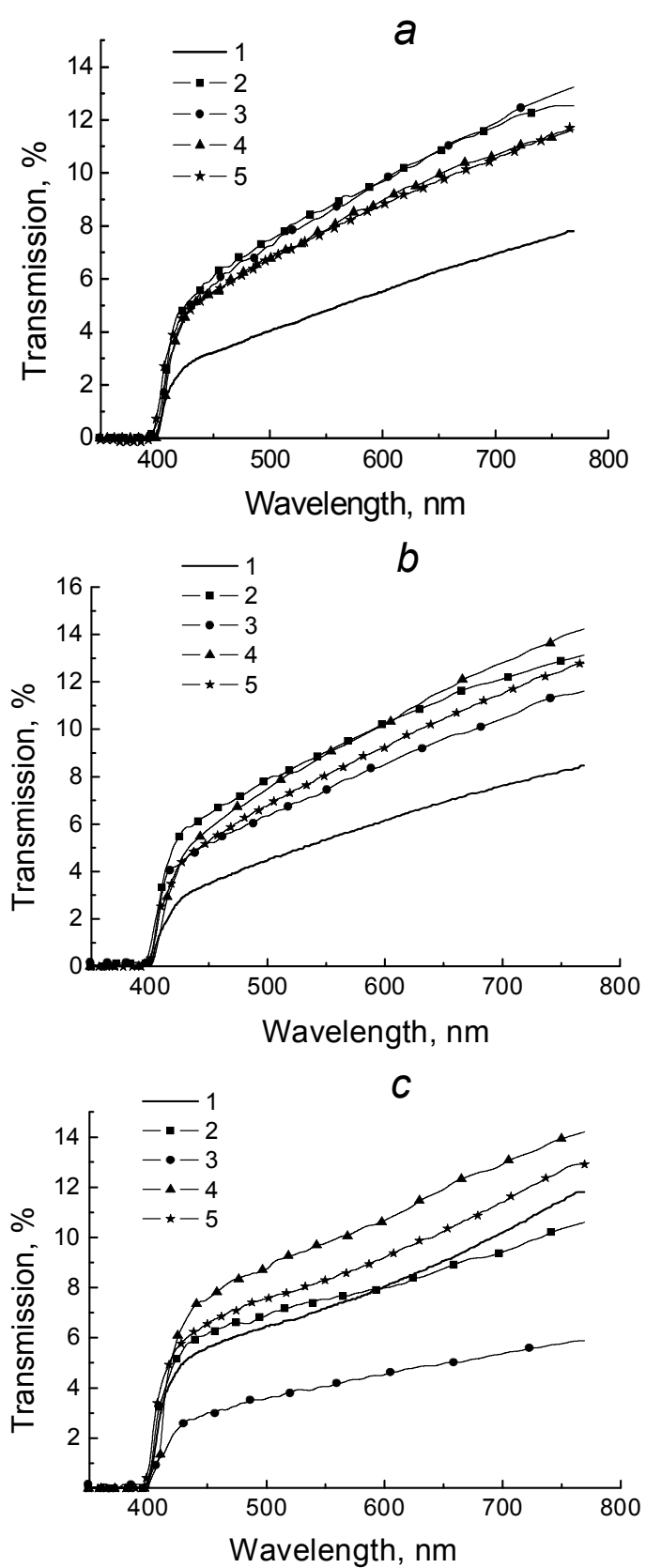

Fig. 1. Transmission spectra of the oxide film/ $\mathrm{SiC}$ structures: (a) $\mathrm{TiO}_{2}$, (b) $\mathrm{Gd}_{2} \mathrm{O}_{3}$, (c) $\mathrm{Er}_{2} \mathrm{O}_{3}$ taken before (1 - initial structure) and after microwave annealing. Total time of microwave annealing: $1 \mathrm{~s}(2), 2$ (3), 3 (4), 8 (5) [6].

To determine the degree of thermal action of microwave radiation on the specimens under investigation, let us estimate temperature variation $\Delta T$ owing to such action. Since the oxide layer does not absorb microwave radiation [13], the only source of specimen heating is absorption of microwave radiation in semiconductor. Let us assume that the specimen absorbs the total microwave radiation, and the absorbed energy is uniformly distributed over the specimen volume. Then the highest possible temperature of heated specimen is
$\Delta T=\frac{E}{V C \rho}$.

Here $V$ is the specimen volume (in our case. the average specimen volume $\left.V=0.0125 \mathrm{~cm}^{3}\right) ; E=W \times t$ is the energy passing to the specimen in a time $t, W=P \times V$, $P$ is the microwave radiation power per specimen unit volume $\left(0.04 \mathrm{~W} / \mathrm{cm}^{3}\right) ; C(\rho)$ is $\mathrm{SiC}$ thermal capacity (density): $C=620-750 \mathrm{~J} / \mathrm{kg} \cdot \mathrm{deg}=0.62-0.75 \mathrm{~J} / \mathrm{g} \cdot \mathrm{deg}$ [14], $\rho=3170 \mathrm{~kg} / \mathrm{m}^{3}=3.170 \mathrm{~g} / \mathrm{cm}^{3}[14]$.

After inserting the above parameters to Eq. (1), taking irradiation time to equal $1 \mathrm{~s}$ and assuming that the total microwave radiation is absorbed by the specimen, we obtain that its temperature may change by $\Delta T=0.02$. Therefore, it is possible to neglect the contribution from thermal mechanism when explaining the observed variations of properties of metal oxide film and silicon carbide at the film/ $\mathrm{SiC}$ interface appearing under microwave irradiation.

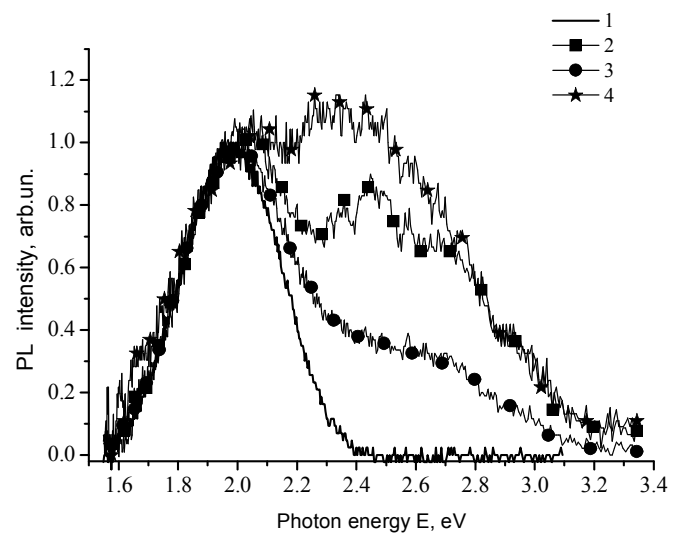

Fig. 2. PL spectra of the oxide film/ $\mathrm{SiC}$ structures taken before $(1$ - initial structure) and after microwave annealing (2 $\left.\mathrm{TiO}_{2} / \mathrm{SiC}, 3-\mathrm{Gd}_{2} \mathrm{O}_{3} / \mathrm{SiC}, 4-\mathrm{Er}_{2} \mathrm{O}_{3} / \mathrm{SiC}\right)$. Total time of microwave annealing $8 \mathrm{~s}$ [6].

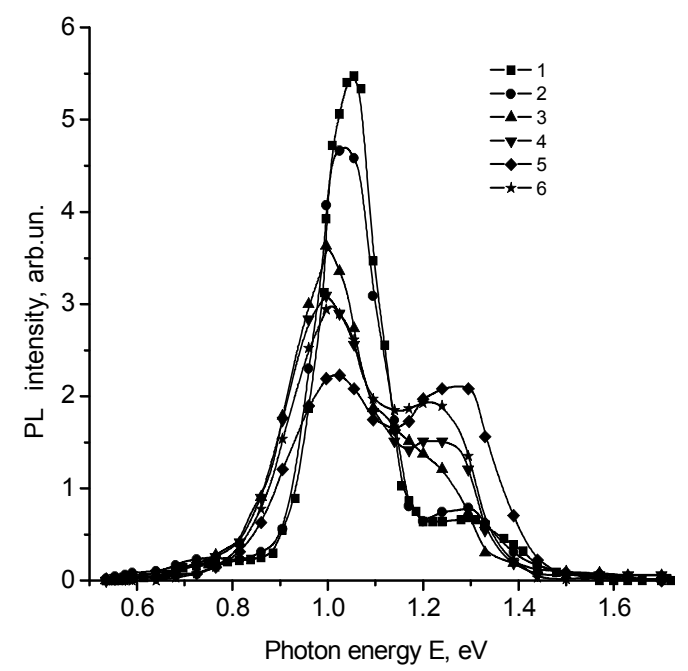

Fig. 3. $\mathrm{PL}$ spectra of the $\mathrm{SiO}_{2} / \mathrm{GaAs}$ structure taken on the side of $\mathrm{SiO}_{2}$ film before ( 1 - initial structure) and after microwave annealing (2-6). Total time of microwave irradiation: $1 \min (2), 2$ (3), 3 (4), 8 (5), 13 (6) [7]. 


\section{Possible model for non-thermal action of microwave radiation}

A single quantum of microwave radiation of frequency $2.45 \mathrm{GHz}$ is $\sim 10^{-5} \mathrm{eV}$. This is much below the activation energy of migration for migration of inherent atoms, their vacancies and impurity atoms in $\mathrm{SiC}$ (from $1.47 \mathrm{eV}$ [4] to $8 \mathrm{eV}$ [15]) as well as phonon energy in silicon carbide $\left(\sim(10-100) \cdot 10^{-3} \mathrm{eV}[16]\right)$.

The most likely reason for appearance of nonthermal effect of microwave radiation is presence in a crystal of nonequilibrium states that cause sensitivity of defect structure to action of microwave radiation. In particular, it was noted in $[17,18]$ when considering effect of low electric fields on nonmagnetic crystals that such effect is just due to presence of structural defects in them. It may be assumed that microwave field (as magnetic field) can lower the potential barriers (related to random distribution of the fields of intrinsic stresses in a crystal) that must be surmounted to provide dislocation movement $[17,18]$.

Besides, microwave radiation can affect not only on the process of dislocation interaction with an obstacle but on the structure of dislocation nuclei and obstacles as well. The obstacles may be several impurity atoms and cation vacancies as well as be of variable composition $[17,18]$. Relaxation of intrinsic stresses leads to inverse effect, namely, gradual decrease of the number of moving dislocations.

In [19] the Frank-Read mechanism of dislocation multiplication was considered to explain the results of action of microwave radiation on GaAs. Special attention was paid to analysis of variation of the critical field strength $E_{c}$ leading to generation of dislocation loops. The calculations made in [19] showed that production of critical electric field $\left(E_{c}=1.7 \times 10^{7} \mathrm{~V} / \mathrm{m}\right)$ in GaAs requires application of microwave frequencies of about $84 \mathrm{GHz}$. At the same time, the authors of [19] considered a possibility of $E_{c}$ reduction as the frequency of microwave oscillations is approaching the eigenfrequencies of dislocation vibrations. For GaAs, the minimal eigenfrequency of vibrations of a dislocation segment of length $L=10^{3} b$ ( $b$ is the Burgers vector) calculated within the string model was $\omega_{1}=13 \mathrm{GHz}$. However, it was noted in [19] that the impurity atoms accumulated at dislocations may lead to decrease of the eigenfrequencies of dislocation vibrations.

Let us consider resonance interaction of microwave radiation with dislocations in somewhat different aspect. Because of lattice mismatch of different layers, elastic stresses appear in substrate and oxide layer of a multilayer system (in our case, the composition of oxide layers is close to stoichiometric one $[4,6])$. Compensation of lattice mismatch of the substrate and oxide layer becomes energetically efficient not only due to elastic strain over the whole interfacial area between the two lattices but partially owing to dislocations appearing at that surface as well [20]. In particular, dislocations in silicon carbide are nuclei of stacking faults (cubic SiC interlayers in $4 \mathrm{H}-\mathrm{SiC}$ or $6 \mathrm{H}-\mathrm{SiC}$ ) in the bulk of epitaxial layer or at the epitaxial layer-substrate interface [21]. In this case, dislocations may split into partials, thus leading to reduction of the energy of elastic lattice distortions around dislocations.

It was noted in [22] that dislocation interaction with a phonon leads to appearance of a stress field that may cause movement of dislocation as a whole. Besides, according to [17], interaction of dislocation with an obstacle (the nature of the obstacle is not considered here) results in appearance, along with characteristic frequencies of the phonon spectrum $\left(v_{p h}=10^{6}-10^{13} \mathrm{~Hz}\right)$, of a set of natural vibrations of dislocations.

According to [23], an equation for displacement $u(y, t)$ of a dislocation loop (of length $l$ ) with rigid ends and vibrating (similarly to an elastic string) under a periodic external action may be presented as

$m_{l} \ddot{u}+B \dot{u}-\gamma \frac{\partial^{2} u}{\partial y^{2}}=\sigma b$.

Here $m_{l}$ is the dislocation effective mass per unit length, $\gamma$ is the effective dislocation line tension, $\sigma=$ $\sigma_{0} e^{i \omega t}$ is the oscillating shear tension caused by an external action, $b$ is the Burgers vector modulus. The boundary conditions are $u(0, t)=u(l, t)=0$. A parameter $B$ in Eq. (2) corresponds to damping constant. The term $\gamma \frac{\partial^{2} u}{\partial y^{2}}$ characterizes restoring force per unit length. The $\gamma$ value is estimated from the relation

$\gamma \sim \frac{1}{2} G b^{2}$

where $G$ is the shear modulus. The quantity $m_{l}$ is determined as

$m_{l} \approx \rho b^{2}$,

where $\rho$ is the material density.

According to [23], the dependence $u(y, t)$ for a dislocation loop is

$u(y, t)=A \sigma_{0}\left(l y-y^{2}\right) e^{i \omega t}$,

where $A=\sigma / 2 \gamma$. By inserting Eq. (5) in Eq. (2) and integrating from $y=0$ to $y=l$, we obtain

$-\omega^{2} m_{l} \frac{A l^{3}}{6}+i \omega B \frac{A l^{3}}{6}+2 \gamma A l=b$,

or

$A=\frac{b}{2 \gamma}\left[1+i \omega \tau-\frac{\omega^{2}}{\omega_{0}^{2}}\right]^{-1}$

where

$\omega_{0}^{2}=\frac{12 \gamma}{m_{l} l^{2}}$ 
and

$\tau=\frac{B l^{2}}{12 \gamma}$.

At low attenuation, the quantity

$v_{0}=\omega_{0} / 2 \pi$

acts as resonance frequency at which $|A|$ achieves its maximal value that is limited by the damping constant only. By inserting Eqs. (3) and (4) in Eq. (8), we obtain for the frequency $\omega_{0}$ :

$\omega_{0}^{2}=\frac{12 \cdot \frac{1}{2} G b^{2}}{\rho b^{2} l^{2}}=\frac{6 G}{\rho l^{2}}$.

Based on Eq. (10), it is possible to estimate size of dislocations for which the frequency $v=2.45 \mathrm{GHz}$ of microwave radiation used in our experiments is resonance. In the case of $v=v_{0}$, we obtain

$l^{2}=\frac{3}{2} \frac{G}{\pi^{2} \rho v_{0}^{2}}$.

Inserting the values of $\rho$ and $G$ for silicon carbide ( $G=160 \mathrm{GPa}$ [14]) and $\mathrm{SiO}_{2}, \mathrm{TiO}_{2}, \mathrm{Gd}_{2} \mathrm{O}_{3}$ and $\mathrm{Er}_{2} \mathrm{O}_{3}$ oxide films in Eq. (12), we obtain that the frequency $v=$ $2.45 \cdot 10^{9} \mathrm{~s}^{-1}$ is resonance for partial dislocations of length $l \approx 10^{-4} \mathrm{~cm}$.

Thus, resonance interaction of $2.45 \mathrm{GHz}$ microwave radiation with dislocations can result in release of dislocations with $l \approx 10^{-4} \mathrm{~cm}$ and their movement in both silicon carbide substrate and oxide layer. The movement of dislocations, in its turn, will lead to variation of distribution of intrinsic stresses in the structure under investigation, with further change of number and configuration of dislocations.

When considering resonance interaction of microwave radiation with dislocations one should take into account that any impurities in a crystal that can serve as obstacles for dislocations are sources of vibrations with frequencies $v_{p h}=10^{6}-10^{13} \mathrm{~Hz}$. Since the frequency of microwave radiation used in the experiment lies in the frequency range of obstacles vibrations, the condition of resonance release of dislocation from an obstacle may be fulfilled not only for dislocations of strictly determined size but also for those of arbitrary size but attached to an obstacle that is vibrating with the resonance frequency.

According to $[20,24]$, presence of dislocations in material leads to local variations of both bandgap width and concentration of impurities and lattice defects (in particular, the stacking faults) near dislocations. In silicon carbide, the stacking faults are interlayers of cubic $\mathrm{SiC}$ in $4 \mathrm{H}-$ or $6 \mathrm{H}-\mathrm{SiC}$ [21]. Owing to presence of free or unsaturated bonds at dislocation nucleus as well as to interaction of dislocations with impurities and lattice defects, the corresponding energy levels appear in the crystal bandgap. As a result, the band structure near dislocations is extremely complicated [24].

Generally a dislocation forms isolated centers that may serve as centers of radiative as well as non-radiative electron-hole recombination [24]. It should be noted that the energy released at non-radiative recombination of an electron-hole pair in $\mathrm{SiC}$ is sufficient for overcoming a barrier preventing atom displacement to another position. I.e. a local reconstruction of hexagonal polytype lattice to that of cubic one occurs, with formation of a cubic polytype interlayer [21].

Anisotropy of dislocation movement [20, 24, 25] enables one to explain the following experimental fact: At similar microwave annealing conditions, structural variations at the macroscopic level (in particular, those in transmission spectra) were detected only if an oxide film was deposited onto a crystalline substrate. No changes in the transmission spectra of a structure were observed after action of microwave radiation if an oxide film was deposited onto a glass substrate. This result may be explained as follows. Because of anisotropy of dislocation movement $[24,25]$, a set of dislocations of preferred orientation appears in a crystalline structure. This results in anisotropic distribution of absorption centers interacting with dislocations that shows itself in the absorption spectra. There are no preferred orientations in glass; therefore, average distribution of defects and dislocations in a glass substrate remains invariable at the macrolevel [26], even if variations in dislocation distribution occur at the microlevel.

\section{Conclusion}

Thus, within the assumption of resonance interaction of microwave radiation with dislocations that leads to variation of dislocation number and configuration, one can conclude that action of microwave radiation has to result in redistribution of recombination centers at the semiconductor-oxide layer interface. This, in its turn, leads to appearance of additional bands in PL spectra of the oxide film $/ \mathrm{SiC}$ structures or intensity redistribution for some bands in PL spectra of the $\mathrm{SiO}_{2} / \mathrm{GaAs}$ structure, as well as to optical density variation for the oxide film/ $\mathrm{SiC}$ structures. It should be taken into account that, because of anisotropy of dislocation movement in crystals, the resonance interaction of microwave radiation with dislocations is most efficient in structures with crystalline substrates.

\section{Acknowledgement}

The author is indebted to Prof. A.M. Svetlichnyi for his interest in this work and valuable discussions.

\section{References}

1. E.D. Atanassova, A.E. Belyaev, R.V. Konakova, P.M. Lytvyn, V.V. Milenin, V.F. Mitin, V.V. Shynkarenko, Effect of Active Actions on the 
Properties of Semiconductor Materials and Structures. NTC "Institute for Single Crystals", Kharkiv, 2007.

2. A.E. Belyaev, E.F. Venger, I.B. Ermolovich, R.V. Konakova, P.M. Lytvyn, V.V. Milenin, I.V. Prokopenko, G.S. Svechnikov, E.A. Soloviev, L.I. Fedorenko, Effect of Microwave and Laser Radiations on the Parameters of Semiconductor Structures. Intas, Kyiv, 2002.

3. Yu.Yu. Bacherikov, R.V. Konakova, E.Yu. Kolyadina, A.N. Kocherov, O.B. Okhrimenko, A.M. Svetlichnyi, Effect of microwave radiation on optical transmission spectra in $\mathrm{SiO}_{2} / \mathrm{SiC}$ structures // Semiconductor Physics, Quantum Electronics \& Optoelectronics, 5(4), p. 391-394 (2002).

4. Yu.Yu. Bacherikov, R.V. Konakova, A.N. Kocherov, P.M. Lytvyn, O.S. Lytvyn, O.B. Okhrimenko, A.M. Svetlichnyi, Effect of microwave annealing on silicon dioxide/silicon carbide structures // Technical Phys., 48(5), p. 598-601 (2003).

5. O.B. Okhrimenko, Methods of improvement of properties of the oxide layer-silicon carbide interface // Ukr. J. Phys. Reviews, 6(1), p. 3-10 (2010).

6. Yu.Yu. Bacherikov, R.V. Konakova, V.V. Milenin, O.B. Okhrimenko, A.M. Svetlichnyi, V.V. Polyakov, Changes in characteristics of gadolinium, titanium, and erbium oxide films on the $\mathrm{SiC}$ surface under microwave treatment // Semiconductors, 42(7), p. 868-872 (2008).

7. O.B. Okhrimenko, Effect of microwave irradiation on radiating centers in the $\mathrm{SiO}_{2} / \mathrm{GaAs}$ structures // Petersburg J. Electronics, 42(1), p. 27-30 (2005), in Russian.

8. L.M. Kustov, I.M. Sinev, Microwave activation of catalysts and catalytic processes // Russian J. Phys. Chemistry A, 84(10), p. 1676-1694 (2010).

9. V.A. Bolotov, Yu.D. Chernousov, E.I. Udalov, Yu.Yu. Tanashev, V.N. Parmon, The features of performing high-temperature chemical reactions under microwave field // Vestnik NGU. Ser. Fizika, 4(2), p. 78-83 (2009), in Russian.

10. J. Jacob, L.H.L. Chia, F.Y.C. Boey, Review. Thermal and non-thermal interaction of microwave radiation with materials // J. Materials Sci., 30, p. 5321-5327 (1995).

11. K.I. Rybakov, A.G. Eremeev, S.V. Egorov, Yu.V. Bykov, Z. Pajkic, M. Willert-Porada, Effect of microwave heating on phase transformations in nanostructured alumina // J. Phys. D: Appl. Phys. 41(10), 102008 (2008).

12. K.I. Rybakov, V.E. Semenov, Nonthermal action of microwaves upon transport processes in ionics (effects, mechanisms, and verification), in: Proc.
Intern. Symp. on Microwave, Plasma and Thermochemical Processing of Advanced Materials. Eds. S. Miyake, M. Samandi, p. 20-25, JWRI, Osaka (1997).

13. Yu.K. Kovneristy, I.Yu. Lazareva, A.A. Ravayev, Materials Absorbing Microwave Radiations. Nauka, Moscow, 1982 (in Russian).

14. Electronic archive New Semiconductor Materials. Characteristics and Properties http://www.ioffe.ru/SVA/NSM/

15. D.V. Kulikov, Yu.V. Trushin, P.V. Rybin, V.S. Kharlamov, Physical model for the evolution of the defect system of silicon carbide with allowance for the internal elastic stress fields during implantation of $\mathrm{Al}^{+}$and $\mathrm{N}^{+}$and subsequent annealing // Technical Phys., 44(10), p. 1168-1174 (1999).

16. Li Xiang-Biao, Shi Er-Wei, Chen Zhi-Zhan, Xiao Bing, Optical characterization of $4 \mathrm{H}-, 6 \mathrm{H}-$ and 15R-SiC crystals // Chinese J. Struct. Chem. 26(10), p. 1196-1202 (2007).

17. Yu.I. Golovin, Magnetoplastic effects in solids // Phys. Solid State, 46(5), p. 789-824 (2004).

18. R.B. Morgunov, Spin micromechanics in the physics of plasticity // Physics-Uspekhi, 47(2), p. 125-147 (2004).

19. I.B. Ermolovich, G.V. Milenin, V.V. Milenin, R.V. Konakova, R.A. Red'ko, Modification of the defect structure in binary semiconductors under the action of microwave radiation // Technical Phys., 52(9), p. 1173-1177 (2007).

20. A.G. Zaluzhnyi, Dislocations in Crystals, Their Movement and Elastic Properties. MIFI, Moscow, 1990 (in Russian).

21. O.A. Ageev, A.E. Belyaev, N.S. Boltovets, V.S. Kiselev, R.V. Konakova, A.A. Lebedev, V.V. Milenin, O.B. Okhrimenko, V.V. Polyakov, A.M. Svetlichnyi, D.I. Cherednichenko, Silicon Carbide: Technology, Properties, Application. "ISMA", Kharkiv, 2010 (in Russian).

22. J.M. Ziman, Electrons and Phonons. Clarendon Press, Oxford, 1960.

23. A.S. Nowick, B.S. Berry, An Elastic Relaxation in Crystalline Solids. Academic Press, New York, 1972.

24. H.F. Matare, Defect Electronics in Semiconductors. Wiley-Interscience, New York, 1971.

25. J.P. Hirth, J. Lothe, Theory of Dislocations. McGraw-Hill, 1967.

26. E. Atanassova, R.V. Konakova, V.F. Mitin, J. Koprinarova, O.S. Lytvyn, O.B. Okhrimenko, V.V. Schinkarenko, D. Virovska, Effect of microwave radiation on the properties of $\mathrm{Ta}_{2} \mathrm{O}_{5}-\mathrm{Si}$ microstructures // Microelectronics Reliability, 45, p. $123-135$ (2005). 